\title{
Surface Sticking and Lateral Diffusion of Lipids in Supported Bilayers
}

\author{
Thomas Dertinger, ${ }^{\dagger}$ Iris von der Hocht, ${ }^{\dagger}$ Aleš Benda, ${ }^{\ddagger}$ Martin Hof,,${ }^{\dagger}$ and Jörg Enderlein*, ${ }^{\dagger}$ \\ Institute for Biological Information Processing I, Forschungszentrum Jülich, D-52425 Jülich, Germany, \\ and J. Heyrovský Institute of Physical Chemistry, Academy of Sciences of the Czech Republic, \\ Dolejškova 3, 18223 Praha 8, Czech Republic
}

Received May 17, 2006. In Final Form: August 19, 2006

\begin{abstract}
The diffusion of fluorescently labeled lipids in supported bilayers is studied using two different methods: Z-scan fluorescence correlation spectroscopy (z-scan FCS) and two-focus fluorescence correlation spectroscopy (2f-FCS). It is found that the data can be fitted consistently only when taking into account partial sticking of the labeled lipids to the supporting glass surface. A kinetic reaction-diffusion model is developed and applied to the data. We find a very slow sticking rate which, however, when neglected, leads to strongly varying estimates of the free diffusion coefficient. The study reveals a strong sensitivity of FCS on even slight binding/unbinding kinetics of the labeled molecules, which has significance for related diffusion measurements in cellular lipid membranes.
\end{abstract}

\section{Introduction}

After the first successful preparation of supported phospholipid bilayers (SPBs) from vesicle suspensions, ${ }^{1}$ their investigation has experienced a tremendous renaissance. They have been intensively studied as model systems for biological membranes and for their potential application in biosensors. ${ }^{2,3}$ A particular topic of interest is the measurement of diffusion of lipids or incorporated proteins in such bilayers, giving information on the phase state of the lipid and on the mobility of incorporated molecules. A particular technique that has found broad application in these studies is fluorescence correlation spectroscopy (FCS). ${ }^{4-7}$ In FCS, a laser beam is tightly focused onto the bilayer, and the in and out diffusion of fluorescently labeled molecules (either lipids or proteins) is monitored by recording the resulting fluorescence intensity fluctuations. ${ }^{8}$ Applying an autocorrelation analysis to the recorded signal yields the so-called autocorrelation function $(\mathrm{ACF})$, showing a characteristic temporal decay which is directly related to the diffusion coefficient of the labeled molecules diffusing within the bilayer. A challenge in common FCS is the determination of precise quantitative values of the diffusion coefficient, which sensitively depends on the exact knowledge of the diameter of the Gaussian intensity profile of the laser focus within the plane of the bilayer. Due to the large beam divergence of a focused laser beam, and the difficulty to exactly locate the bilayer's position relative to the laser's beam waist, it is usually difficult to obtain exact knowledge on focus diameter within the plane of the bilayer. Recently, Benda et al. have found an elegant solution for this problem: By repeating the measurement at different relative vertical positions of the laser beam with respect to the bilayer ( $z$-scan-FCS), they were

* Corresponding author. Fax: +49-2461 614216; E-mail: j.enderlein@ fz-juelich.de.

Forschungszentrum Jülich.

$\doteqdot$ Academy of Sciences of the Czech Republic.

(1) Brian, A. A.; McConnell, H. M. Proc. Nat. Acad. Sci. 1984, 81, 61596163

(2) Sackmann, E. Science 1996, 271, 43-48.

(3) Boxer, S. G. Curr. Opin. Chem. Biol. 2000, 4, 704-709.

(4) Burns, A. R.; Frankel, D. J.; Buranda, T. Biophys. J. 2005, 89, 1081-1093.

(5) Zhang, L.; Granick, S. Proc. Nat. Acad. Sci. 2005, 102, 9118-9121.

(6) Zhang, L.; Granick, S. J. Chem. Phys. 2005, 123, 211104.

(7) Hac, A. E.; Seeger, H. M.; Fidorra, M.; Heimburg, T. Biophys. J. 2005, $88,317-333$

(8) Rigler, R.; Elson, E., Eds. Fluorescence Correlation Spectroscopy; Springer: New York, 2001. able to determine the quantitative diffusion coefficient within membranes with high accuracy. ${ }^{9}$ Here, we apply a second method which addresses the same problem, namely 2-focus FCS: By measuring the ACFs in two overlapping laser foci and also the cross-correlation between the foci, and using the exact knowledge of the foci distance, it is also possible to extract quantitative values of diffusion coefficients without the necessity to perform a complete $z$-scan. However, the situation becomes much more challenging when, besides free diffusion of the labeled molecules within the bilayer, additional adsorption of the labeled molecules to the bilayer's supporting surface comes into play. In the present paper, we develop the theoretical framework for FCS measurements on such systems, exhibiting free diffusion within a planar bilayer plus adsorption/desorption kinetics to the supporting surface, and apply both $z$-scan and 2-focus FCS for experimentally studying such systems.

\section{Theory}

In the present section, we will consider diffusion of a molecule within a planar layer, which can undergo adsorption and desorption to a surface supporting the layer. The situation and mathematical approach is similar to that found in the literature, ${ }^{10,11}$ where the authors considered three-dimensional diffusion in solution above a surface and adsorption/desorption kinetics to the surface. However, the final result will be quite different, and no closed analytical solution can be derived in our case.

It is assumed that the FCS measurements are performed with focused laser beams having Gaussian intensity profiles with identical $1 / e^{2}$-radius $w$. Then, the fluorescence cross-correlation function $g(t, \delta)$ for the fluorescence cross-correlation between two laterally displaced foci is given by

$$
\begin{array}{r}
g(t, \delta)=\kappa^{2} c_{0} \int \mathrm{d} \mathbf{r} \int \mathrm{d} \mathbf{r}_{0} \exp \left(-\frac{2|\mathbf{r}-\boldsymbol{\delta}|^{2}}{w^{2}}\right) p\left(\mathbf{r}-\mathbf{r}_{0}, t\right) \times \\
\exp \left(-\frac{2 r_{0}^{2}}{w^{2}}\right)+\left(\kappa c_{0}\right)^{2} \int \mathrm{d} \mathbf{r} \exp \left(-\frac{2 r^{2}}{w^{2}}\right)
\end{array}
$$

where $\kappa$ is some constant factor depending on overall detection efficiency, excitation cross section, total laser power, etc., $c_{0}$ is the molecules' two-dimensional concentration, $\boldsymbol{\delta}$ is the distance 
vector between the foci, and $p\left(\mathbf{r}-\mathbf{r}_{0}, t\right)$ denotes the probability density that a molecule moves from position $\mathbf{r}_{0}$ to position $\mathbf{r}$ within time $t$. All integrations are performed over the whole two-dimensional surface. It should be noticed that the final result $g(t, \delta)$ depends only on the absolute value $\delta$, but not on its direction. The result for the autocorrelation is obtained by setting $\delta=0$ in the above expression.

Thus, the problem reduces to finding an expression for $p(\mathbf{r}-$ $\left.\mathbf{r}_{0}, t\right)$. Let us denote the probability density to find a freely diffusing molecule at position $\mathbf{r}$ and time $t$ by $a(\mathbf{r}, t)$ and the corresponding probability density to find an adsorbed (bound) molecule at position $\mathbf{r}$ and time $t$ by $b(\mathbf{r}, t)$. The equations governing the temporal evolution of these functions are

$$
\begin{gathered}
\frac{\partial a}{\partial t}=D \Delta a-k_{+} a+k \_b \\
\frac{\partial b}{\partial t}=k_{+} a-k_{-} b
\end{gathered}
$$

where $D$ is the diffusion coefficient, $\Delta$ denotes the twodimensional Laplace operator, and $k_{+}$and $k_{-}$are the adsorption and desorption rate constants of molecule to and from the supporting surface, respectively. Under equilibrium conditions, the probabilities to find a molecule in a freely diffusing or an adsorbed state are $k_{-} /\left(k_{+}+k_{-}\right)$and $k_{+} /\left(k_{+}+k_{-}\right)$, respectively. Thus, $p\left(\mathbf{r}-\mathbf{r}_{0}, t\right)$ can be found as the sum of solutions $a\left(\mathbf{r}-\mathbf{r}_{0}, t\right)$ and $b\left(\mathbf{r}-\mathbf{r}_{0}, t\right)$ of the above system of equations with initial conditions

$$
\begin{aligned}
& a\left(\mathbf{r}-\mathbf{r}_{0}, 0\right)=\frac{k_{-}}{k_{+}+k_{-}} \delta\left(\mathbf{r}-\mathbf{r}_{0}\right) \\
& b\left(\mathbf{r}-\mathbf{r}_{0}, 0\right)=\frac{k_{+}}{k_{+}+k_{-}} \delta\left(\mathbf{r}-\mathbf{r}_{0}\right)
\end{aligned}
$$

Applying a Fourier transform to eqs $2 \mathrm{a}$ and $2 \mathrm{~b}$ leads to the straightforward solution for the Fourier transform of $p$ as

$$
\begin{gathered}
p(\mathbf{q}, t)=\frac{\left(k_{+}+k_{-}\right)\left(k_{+}+k_{-}+\Delta\right)+\left(k_{+}-k_{-}\right) D q^{2}}{2\left(k_{+}+k_{-}\right) \Delta} \exp \\
\left(\omega_{1} t-i \mathbf{q} \cdot \mathbf{r}_{0}\right)- \\
\frac{\left(k_{+}+k_{-}\right)\left(k_{+}+k_{-}-\Delta\right)+\left(k_{+}-k_{-}\right) D q^{2}}{2\left(k_{+}+k_{-}\right) \Delta} \exp \left(\omega_{2} t-i \mathbf{q} \cdot \mathbf{r}_{0}\right)
\end{gathered}
$$

where $\mathbf{q}$ is the Fourier transformed coordinate, and the abbreviations

$$
\begin{gathered}
\omega_{1,2}=-\frac{D q^{2}+k_{+}+k_{-} \mp \Delta}{2}, \\
\Delta_{2}=\left(D q^{2}+k_{+}+k_{-}\right)^{2}-4 D q^{2} k_{-}=\left(D q^{2}+k_{+}-k_{-}\right)^{2}+ \\
4 k_{+} k_{-}(5 \mathrm{~b})
\end{gathered}
$$

were used. Converting eq 4 back to real space, and inserting the result into eq 1 yields the final result for the auto-/cross-correlation function:

(9) Benda, A.; Benes, M.; Marecek, V.; Lhotsky, A.; Hermens, W. T.; Hof, M. Langmuir 2003, 19, 4120-4126.

(10) Lieto, A. M.; Cush, R. C.; Thompson, N. L. Biophys. J. 2003, 85, 32943302 .

(11) Starr, T. E.; Thompson, N. L. Biophys. J. 2001, 80, 1575-1584.

$$
\begin{array}{r}
g(t, \delta)=g_{\infty}+\kappa^{2} c_{0} \int_{0}^{\infty} d q q \\
{\left[\frac{\left(k_{+}+k_{-}\right)\left(k_{+}+k_{-}+\Delta\right)+\left(k_{+}-k_{-}\right) D q^{2}}{2\left(k_{+}+k_{-}\right) \Delta} \exp \left(\omega_{1} t\right)-\right.} \\
\left.\frac{\left(k_{+}+k_{-}\right)\left(k_{+}+k_{-}-\Delta\right)+\left(k_{+}-k_{-}\right) D q^{2}}{2\left(k_{+}+k_{-}\right) \Delta} \exp \left(\omega_{2} t\right)\right] J_{0} \\
(q \delta) \exp \left(-\frac{q^{2} w^{2}}{4}\right)
\end{array}
$$

where $J_{0}$ is Bessel's function of the first kind, ${ }^{12}$ and $g_{\infty}$ denotes the constant offset of the autocorrelation function reached at $t$ $\rightarrow \infty$. In the limit of vanishing adsorption rate constant, i.e., uninhibited free diffusion in a plane, this result reduces to the standard expression

$$
\begin{array}{r}
g(t)=g_{\infty}+\kappa^{2} c_{0} \int_{0}^{\infty} d q q J_{0}(q \delta) \exp \left(-D q^{2} t-\frac{q^{2} w^{2}}{4}\right)= \\
g_{\infty}+\frac{2 \kappa^{2} c_{0}}{4 D t+w^{2}} \exp \left(-\frac{\delta^{2}}{4 D t+w^{2}}\right) .
\end{array}
$$

When using eq 6 for fitting our experimental data, the occurring integral was evaluated numerically by using a standard Romberg integration scheme. ${ }^{13}$

\section{Materials and Methods}

Measurement System. There are several reports in the literature on FCS using more than a single excitation focus, mostly for studying directed transport in solution. ${ }^{14,15}$ None of the published work used overlapping foci, due to the technical problem of differentiating between photons excited by the two overlapping lasers. However, focus overlap is important when aiming at measuring the crosscorrelation between two foci caused by diffusion of molecules, because, as shown in the theory section, the amplitude of the crosscorrelation function decays as the exponent of the square of the foci distance, thus making measurements impracticable for foci distances much larger than the foci diameters. Thus, we have developed a new 2-focus confocal epifluorescence microscope for fluorescence correlation studies which is schematically shown in Figure 1. The light of two spectrally identical $(640 \mathrm{~nm})$, linearly polarized, and pulsed lasers (LDH-P-635, PicoQuant GmbH, Berlin, Germany) is combined by a polarizing beam splitter (Narrow Band Polarizing Beam splitter Cube 633, Ealing Catalog, St. Asaph, UK) to yield a single beam with crossed polarizations. This light is sent through a single-mode optical fiber for mode cleaning. After exiting the fiber, the light is recollimated, reflected by a dichroic mirror (Q 660 LP, Chroma Technology, Rockingham, VT) and passed through a differential interference contrast (DIC) prism (U-DICHC, Olympus Europa $\mathrm{GmbH}$, Hamburg, Germany). The prism deflects the light from the two lasers into slightly different directions, so that after focusing through the objective (UPLAPO $60 \times \mathrm{W}, 1.2$ N.A., Olympus Europa GmbH, Hamburg, Germany) one obtains two laterally shifted but overlapping laser beams with parallel propagation axes. The distance between the beams is uniquely defined by the chosen DIC prism and is in our system equal to $400 \mathrm{~nm}$, as measured by scanning the point spread functions with fluorescent beads. Fluorescence excited within the foci of these beams is collected by the same objective, passed through the DIC prism and the dichroic mirror, and focused into a single circular aperture (diameter $200 \mu \mathrm{m}$ ) which

(12) Abramowitz, M.; Stegun, I. A., Eds. Handbook of mathematical functions; Harry Deutsch: Thun and Frankfurt/Main, 1984.

(13) Press: W. H.; Teukolsky, S. A.; Vetterling, W. T.; Flannery, B. P. Numerical recipes in C, 2nd ed.; Cambridge University Press: Cambridge, 1992

(14) Brinkmeier, M.; Dörre, K.; Stephan, J.; Eigen, M. Anal. Chem. 1999, 71, $609-616$.

(15) Lumma, D.; Best, A.; Gansen, A.; Feuillebois, F.; Rädler, J. O. Vinogradova, O. I. Phys. Rev. E 2003, 67, 056313. 


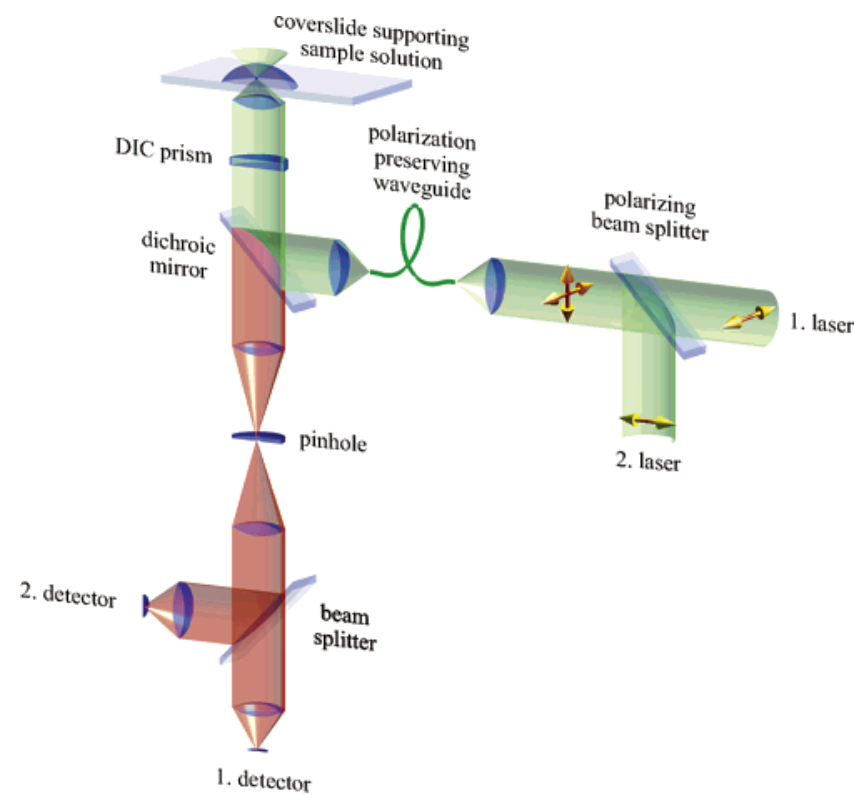

Figure 1. Schematic of the experimental setup showing the optics of the 2 -focus generation.

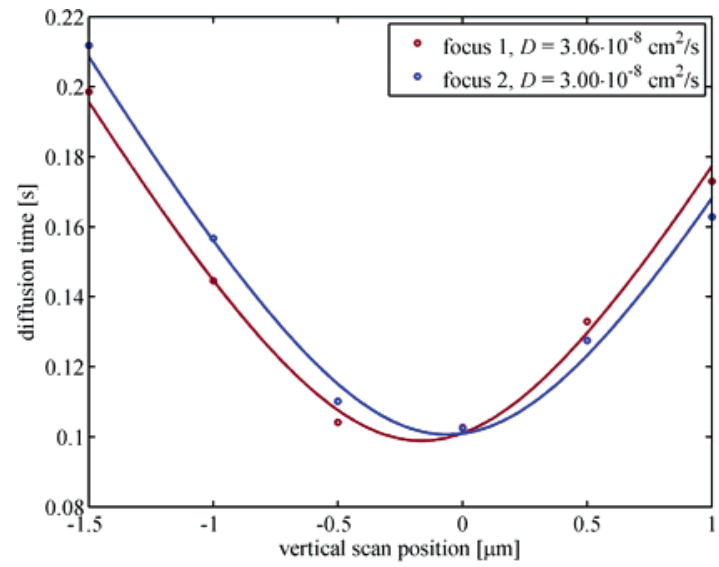

Figure 2. Dependence of diffusion time on vertical scan position for the first (red circles) and second focus (blue circles). Solid lines represent least-squares fits of the data using the scalar approximation of a Gaussian laser beam.

is positioned symmetrically with respect to both focus positions and chosen large enough to let light easily pass from both foci. After the pinhole, the light is recollimated, split by a nonpolarization sensitive beam splitter cube (Linos Photonics GmbH \& Co. KG, Göttingen, Germany), and focused onto two single-photon avalanche diodes (SPCM-AQR-14, Perkin-Elmer, Wellesley, MA). Single photons detected by the avalanche diodes are then processed by fast photon counting electronics (TimeHarp 200, PicoQuant GmbH) capable of both timing the macroscopic arrival time of the photons with $100 \mathrm{~ns}$ temporal resolution and timing the arrival of the same photons with respect to the laser pulses with picosecond temporal resolution (time-correlated single-photon counting or TCSPC).

Both lasers are pulsed alternatively (pulsed interleaved excitation or PIE, ${ }^{16}$ also known as alternating laser excitation or ALEX ${ }^{17}$ ) with $40 \mathrm{MHz}$ overall repetition rate and ca. $50 \mathrm{ps}$ pulse width. Alternative pulsing is accomplished by special laser driver electronics (PDL 808 "Sepia", PicoQuant GmbH). The temporal distance between two subsequent laser pulses (25 ns) was chosen to be much larger than the fluorescence lifetime $(2 \mathrm{~ns})$ of the dye used in the

(16) Müller, B. K.; Zaychikov, E.; Bräuchle, C.; Lamb, D. C. Biophys. J. 2005 , $89,3508-3522$.

(17) Kapanidis, A. N.; Laurence, T. A.; Lee, N. K.; Margeat, E.; Kong, X.; Weiss, S. Acc. Chem. Res. 2005, 38, 523-533.

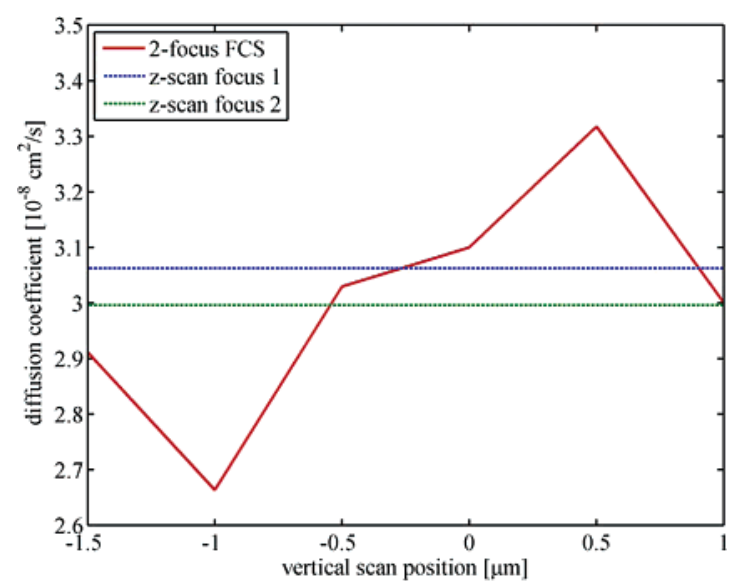

Figure 3. Dependence of diffusion coefficient on vertical scan position as obtained from the 2f-FCS measurements using the freediffusion model. Broken lines indicate the values of the diffusion coefficient as obtained from z-scan analysis (see Figure 2).

measurements, so that it was possible to correlate detected photons to their exciting laser via checking, on a nanosecond time scale, which laser was pulsed last before the detection. This allows us to calculate photon autocorrelations separately for each focus as well as the photon cross-correlation between the two foci, even if both excitation beams are strongly overlapping. Correlation calculations were performed on the basis of the recorded macroscopic arrival times of detected photons as described in ref 18 . Only photons from different detectors were correlated to prevent any impact of detector afterpulsing on the calculated correlation curves. ${ }^{19}$ Vertical position of the objective with respect to the sample was adjusted with nanometer precision using a PiFoc (P-721.00, Physik Instrumente (PI) $\mathrm{GmbH} \& \mathrm{Co}$. KG, Karlsruhe, Germany).

It should be noted that the presence of the DIC prism in the detection channel has negligible effect on the collection efficiency function, which we have checked by detailed wave-optical calculation which will be presented elsewhere: In each plane perpendicular to the optical axis, the two-dimensional molecule detection function in both foci is satisfactorily described by a two-dimensional Gaussian distribution with width $w(z)$ depending on vertical $z$-position. Also, the slightly asymmetric position of the circular aperture with respect to each focus has only a marginal effect on the dependence of the function $w(z)$ on $z$ : For both foci, $w(z)$ can be satisfactorily approximated by the scalar approximation of a Gaussian laser beam (see e.g. ${ }^{20}$ ), i.e., $w(z)=w_{0} \sqrt{1+\left(\lambda z / \pi w_{0}{ }^{2}\right)^{2}}$, where $w_{0}$ is the laser beam waist and $\lambda$ the laser wavelength within the immersion medium (water).

Lipid Layer Preparation. For the measurement of lateral diffusion it is desirable to prepare defect-free SPBs. SPBs can be prepared by Langmuir-Blodgett technique or by vesicle fusion. A rehydration method leads to multilayer structures. ${ }^{21}$ Preparation of defect-free fluid phase SPBs by the LB technique is an extremely difficult task with pure reproducibility and control of the quality of the resulting bilayer. ${ }^{22,23}$ Only the vesicle fusion (with defined experimental conditions-surface cleaning, buffer, lipid composition) was shown by AFM to lead to defects free fluid phase bilayers. ${ }^{24,25}$ Thus, we have chosen this method for SPB preparation.

DOPC (1,2-dioleoyl-sn-glycerophophocholine) was purchased from Avanti Polar Lipids (Alabaster, AL). DOPE (1,2-dioleoyl$s n$-glycero-3-phosphoethanolamine) was purchased from Sigma (St. Louis, MO). Atto655-NHS-ester was purchased from Atto-Tec

(18) Wahl, M.; Gregor, I.; Patting, M.; Enderlein, J. Opt. Expr. 2003, 11, 3583-3591.

(19) Enderlein, J.; Gregor, I. Rev. Sci. Instrum. 2005, 76, 033102.

(20) Enderlein, J.; Pampaloni, F. J. Opt. Soc. Am. A 2004, 21, 1553-1558.

(21) Salditt, T. J. Phys. - Condens. Matter 2005, 17, R287-R314.

(22) Bassereau, P.; Pincet, F. Langmuir 1997, 13, 7003-7007.

(23) Osborn, T. D.; Yager, P. Biophys. J. 1995, 68, 1364-1373.

(24) Reviakine, I.; Brisson, A. Langmuir 2000, 16, 1806-1815.

(25) Richter, R. P.; Brisson, A. Langmuir 2003, 19, 1632-1640. 
(a)
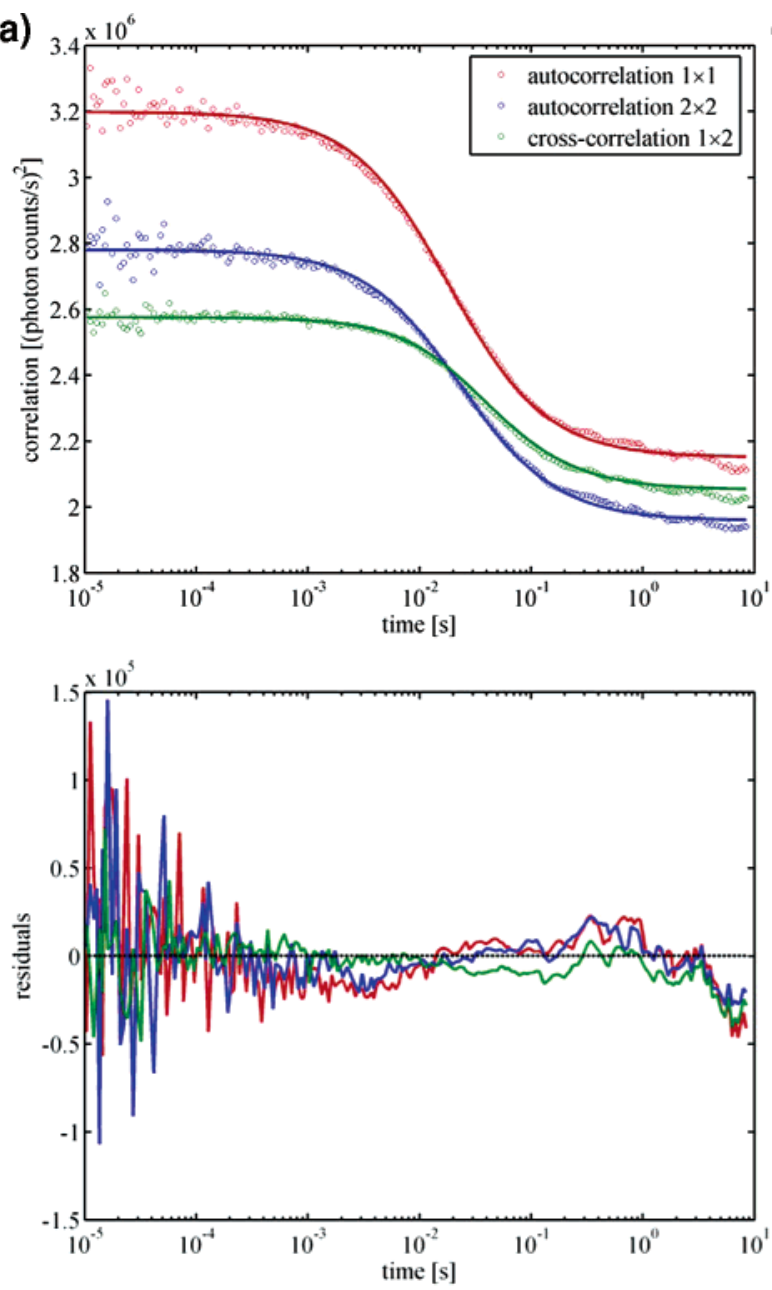

(b)
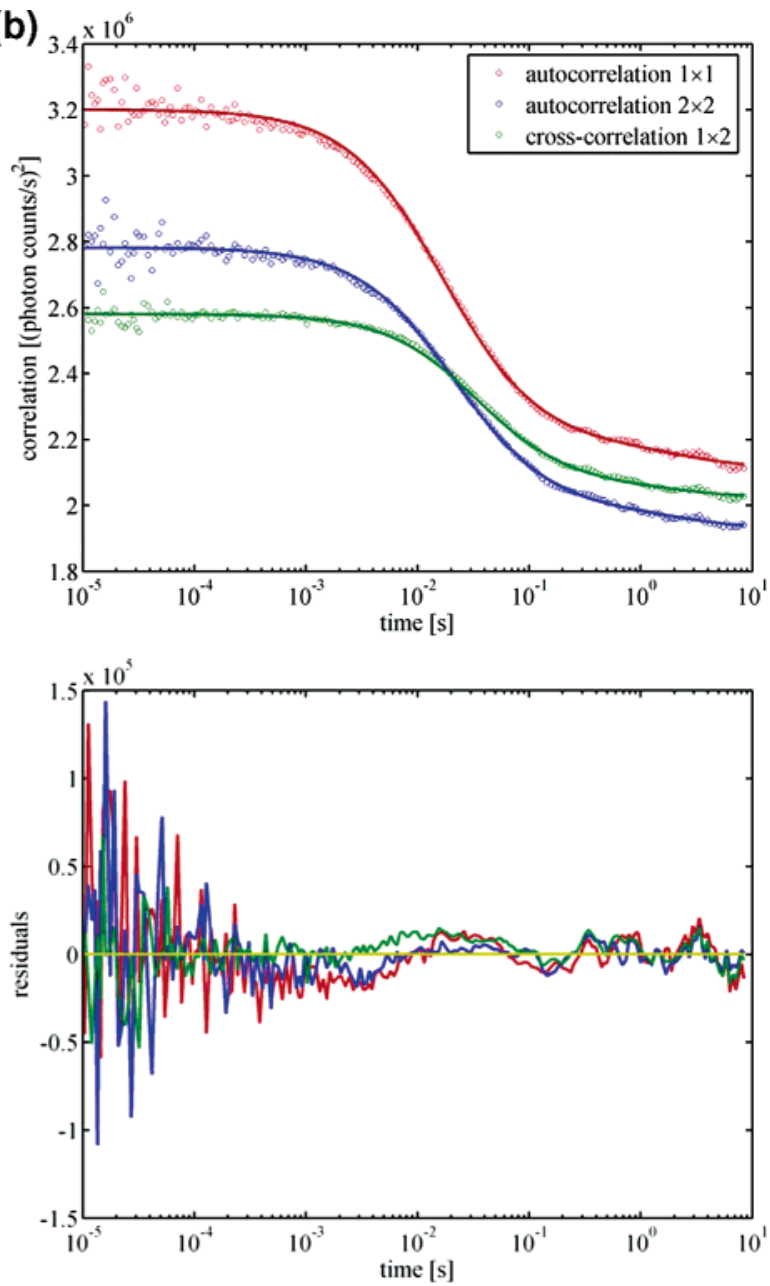

Figure 4. (a) Simultaneous least-squares fit of the two auto- and one cross-correlation curves for one z-position using the free-diffusion model. Upper panel shows the fit, lower panel the residuals. (b) Same as part a but using model eq 6 including adsorption and desorption kinetics.

GmbH (Siegen, Germany). All other chemicals were purchased from Sigma, Fluka (St. Gallen, Switzerland) and kmf Laborchemie Handels GmbH (Lohmar, Germany).

To label the headgroups of the phospholipids with the fluorescent dye, $1.14 \mu \mathrm{mol}$ DOPE, $1.14 \mu \mathrm{mol}$ triethylamine, and $1.6 \mu \mathrm{mol}$ Atto655-NHS-ester were dissolved in $60 \mu \mathrm{L}$ anhydrous methanol and incubated for $90 \mathrm{~min}$ at room temperature. Reaction progress was followed by thin-layer chromatography using silica gel $60-\mathrm{F}_{254}$ plates (Merck, Darmstadt, Germany). The plates were developed with a 60:25:4 (v/v) mixture of chloroform:methanol:water. Atto655DOPE was purified by chromatography on a silica gel column (eluent: chloroform:methanol:water 60:25:4 (v/v)). The presence of Atto655-DOPE in each fraction was monitored by thin-layer chromatography. The solvent was removed and Atto655-DOPE was dissolved in anhydrous methanol and stored at $-20^{\circ} \mathrm{C}$ under a nitrogen atmosphere until use.

DOPC was dissolved in chloroform. The solutions were handled in glassware only and stored at $-20^{\circ} \mathrm{C}$ under a nitrogen atmosphere. A mixture of labeled and unlabeled lipids was made by mixing appropriate amounts of lipid solutions (labeling ratio 1:400000). The solvent was evaporated under reduced pressure. The sample was kept in a vacuum for additional 45 min to remove remaining solvent. The lipid-film was hydrated with double distilled water. Vesicles were produced by sonification to clarity, during which the solution was kept in an ice-bath. For sonification the tip-sonicator Sonifier Cell Disrupter B12 (Branson, Danbury, CT) was used, yielding a solution of vesicles with a diameter of $80-100 \mathrm{~nm}$ (verified by dynamic light scattering). The lipid concentration in the vesicular suspension was $500 \mu \mathrm{M}$. Metal particles originating from the sonicator tip were removed by centrifugation.
Borosilicate glass cover slides (Menzel $\mathrm{GmbH}+\mathrm{Co} \mathrm{KG}$, Braunschweig, Germany) were cleaned with freshly prepared piranha solution $\left(30 \% \mathrm{H}_{2} \mathrm{O}_{2}\right.$ and concentrated $\mathrm{H}_{2} \mathrm{SO}_{4}$ ratio 2:3), washed extensively with water, and dried in a stream of nitrogen. To build a supported bilayer on the glass slide by vesicle fusion, the vesicle suspension was deposited on the glass slide and incubated for $3 \mathrm{~min}$ at room temperature. Redundant vesicles were washed away. The resulting membrane was never exposed to air. The formation of a continuous supported bilayer under these conditions was checked by fluorescence microscopy imaging and fluorescence recovery after photobleaching (FRAP).

\section{Results and Discussion}

Fluorescence was measured on supported lipid bilayers at different vertical positions of the objective, thus obtaining autocorrelation and cross-correlation curves for different relative positions of the diverging laser beams with respect to the layer (z-scan). Each measurement lasted for ca. $15 \mathrm{~min}$. The autocorrelation curves where then evaluated by applying a $z$-scan analysis as described in ref 9 , assuming that laser beam diameter as a function of vertical position is well described by the scalar field approximation of a Gaussian laser beam. Thus, each autocorrelation curve was first fitted with the standard model for freely diffusing molecules within a plane (i.e. eq 7 with $\delta=0$ ) using the diffusion time $\omega^{2} / 4 D$ as the fit parameter, and the obtained values of diffusion time $\omega^{2} / 4 D$ as a function of vertical $z$-position were fitted by the function $\left(w_{0}^{2} / 4 D\right)\left\{1+\left[\lambda\left(z-z_{0}\right) /\right.\right.$ $\left.\left.\pi w_{0}^{2}\right]^{2}\right\}$, using beam waist $w_{0}$, focal plane position $z_{0}$, and diffusion 


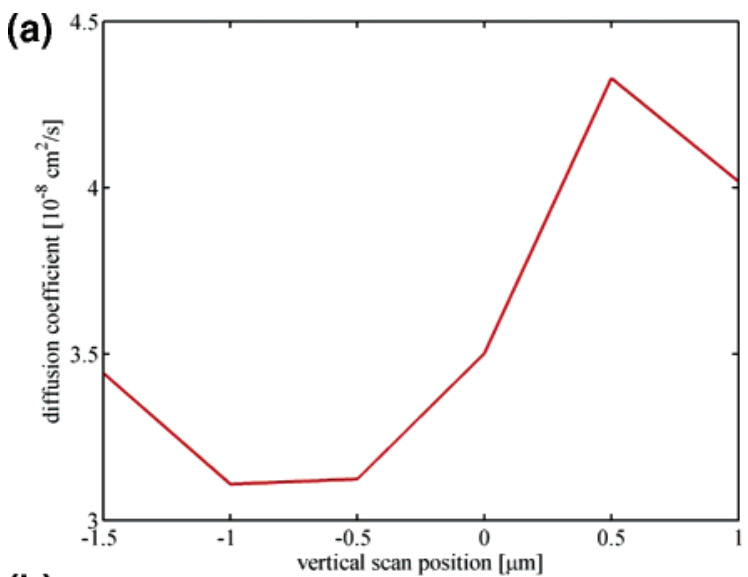

(b)

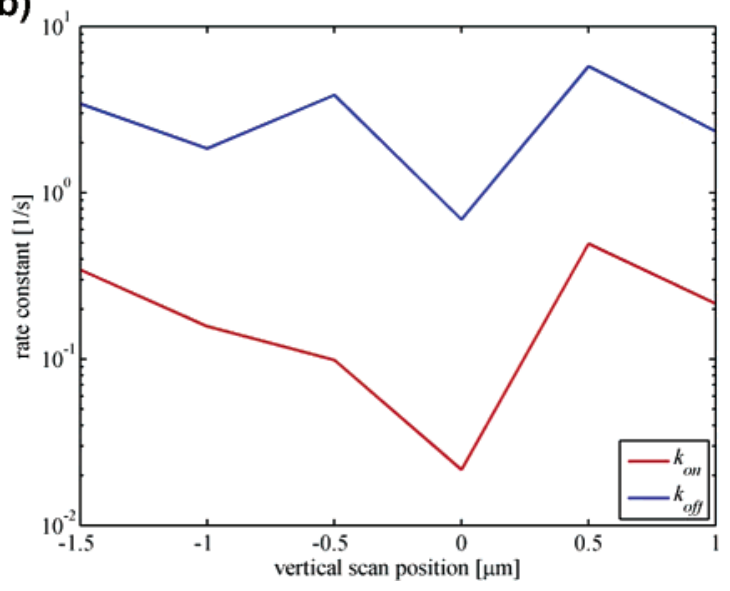

Figure 5. (a) Dependence of diffusion coefficient on vertical scan position as obtained from fitting the $2 \mathrm{f}-\mathrm{FCS}$ measurements using the full model of eq 6. (b) Dependence of adsorption and desorption rate constants on vertical scan position as obtained from fitting the 2fFCS measurements using the full model of eq 6 .

coefficient $D$ as fit parameters. For each focus, the fit of the diffusion time as a function of the vertical position is presented in Figure 2. The obtained values of the diffusion coefficient were $3.00 \times 10^{-8} \mathrm{~cm}^{2} / \mathrm{s}$ and $3.06 \times 10^{-8} \mathrm{~cm}^{2} / \mathrm{s}$, respectively.

Alternatively, we performed a two-focus auto- and crosscorrelation analysis using the calculated auto- and crosscorrelation curves obtained for each vertical focus position. Thus, for each z-position, the two autocorrelation and one crosscorrelation curves were fitted by eq 7 with $\delta=0 \mathrm{~nm}$ and $\delta=$ $400 \mathrm{~nm}$, respectively, using beam waist $w_{0}$ and diffusion coefficient $D$ as fit parameters. It is important to notice that the fit had to reproduce the measured relative ratio of the amplitudes between auto- and cross-correlation, which occurred as a sensitive fit criterion. The relative ratio of the amplitudes is determined by the degree of overlap between the foci, and from the distance $\delta$ between the foci, it is sensitively dependent on the beam waist radius. From the cross-correlation analysis, it was expected to find similar values of the diffusion coefficient as obtained from the single-focus $z$-scan analysis. However, a cross-correlation analysis returns values of the diffusion coefficient separately for each vertical position without the need to perform a full $z$-scan. The obtained values of the diffusion coefficient as a function of vertical z-position are shown in Figure 3, together with an indication of the diffusion coefficients as obtained from the $z$-scan analysis (broken lines). As can be seen, the 2-focus result varies considerably (by more than $15 \%$ around its average) and nonsystematically over all $z$-positions. This large variation can be understood by inspecting the individual fits of the auto- and cross-correlation curves, as shown for one z-position in Figure
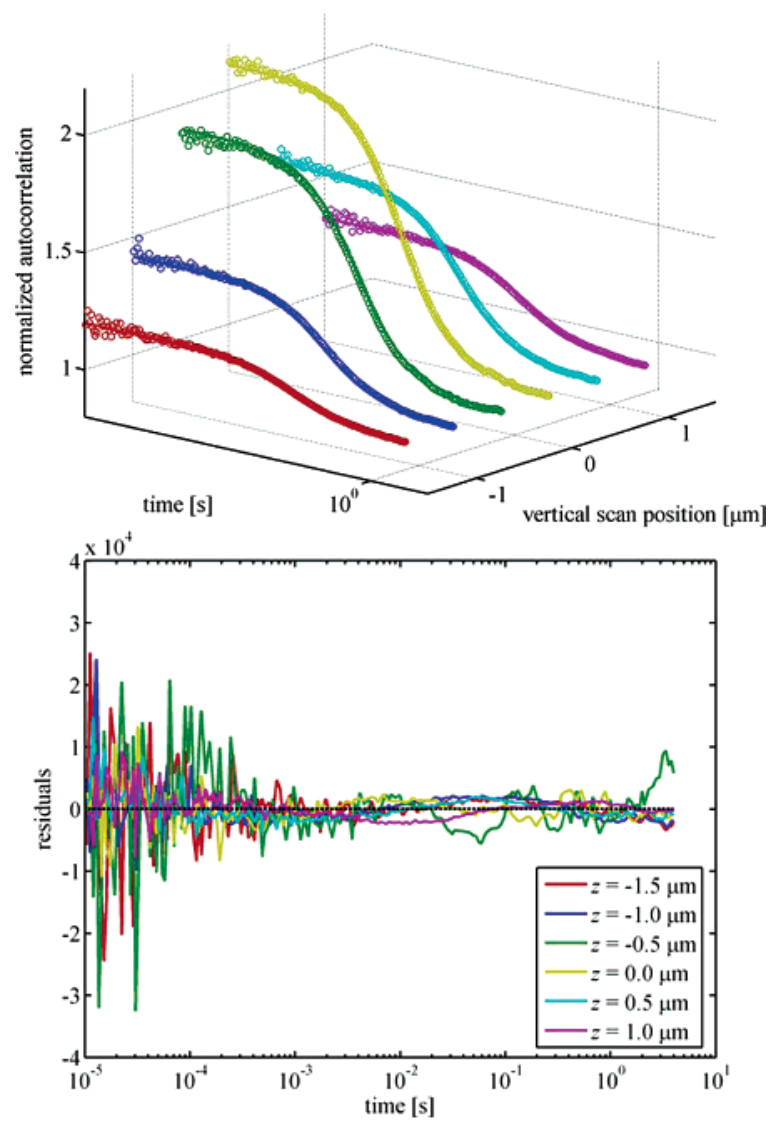

Figure 6. Global fit (solid lines) of the measured autocorrelation curves (circles) for the first focus using eq 6 with $\delta=0$ and one set of parameters assuming that the dependence of laser beam radius on vertical scan position is given by the scalar approximation of a Gaussian laser beam. Upper panel shows the global fit to all curves, lower panel the residuals.

4a. Fit quality is rather poor, in particular at large lag times, and the measured correlations are not well described by the freediffusion-in-two-dimensions model. We attribute that to unspecific adsorption and desorption of labeled lipid molecules to the supporting glass substrate, which was also observed by direct imaging of the samples with single molecule sensitivity. Thus, the analysis of the cross-correlation curves was repeated employing the extended model of eq 6 , now using $w_{0}, D$, and the adsorption and desorption rate constants $k_{+}$and $k_{-}$as fit parameters. The fit result for the same measurement as shown in Figure $4 a$ is presented in Figure $4 b$, showing a clear improvement of fit quality. The resulting values of diffusion coefficient as well as adsorption and desorption rate constants are presented in Figure 5a and 5b. Still, there is considerable variation in all obtained values with varying $z$-position, besides systematically increased values of the diffusion coefficient when compared with the values obtained from the free-diffusion models. An explanation of this strong variation can be found when realizing the slowness of the observed adsorption/desorption kinetics: On average, a molecule adsorbs to the surface ca. every hundred milliseconds, and the desorption kinetics is even slower by more than 1 order of magnitude. Thus, during the measurement time (15 $\mathrm{min}$ ) of one curve only a statistically small number of adsorption and desorption events takes place. Thus, curves measured at different times vary considerably and give strongly varying fit results. Another peculiarity is that the obtained desorption rates are so small that the assumed desorption process is probably rather photobleaching than real desorption with subsequent diffusion out of focus. Extending the measurement 
Table 1.

\begin{tabular}{lcccc}
\hline & $1 / k_{\text {on }}[\mathrm{s}]$ & $1 / k_{\text {off }}[\mathrm{s}]$ & $K_{\text {eq }}=k_{\text {on }} / k_{\text {off }}$ & $D\left[10^{-8} \mathrm{~cm}^{2} / \mathrm{s}\right]$ \\
\hline focus 1 & 0.071 & 2.3 & 0.031 & 3.28 \\
focus 2 & 0.090 & 3.4 & 0.026 & 3.22 \\
2-focus & 0.086 & 2.7 & 0.031 & 3.30
\end{tabular}

time is not a practicable option: To obtain a reasonable statistical accuracy, measurement times of several hours would be needed, ensuring that no change in the sample takes place. To alleviate the situation to some extent, we performed a global fit of all $z$-scan-sets of curves, with one set of parameters $w_{0}, D, k_{+}$, and $k_{-}$for each $z$-scan, and assuming that laser beam radius depends on vertical scan position as described by the scalar approximation of a Gaussian laser beam (similar to the assumption underlying $z$-scan analysis). A typical fit result is shown in Figure 6 for the autocorrelation curves of the first focus. All fit results are listed in Table 1. Employing a global fit approach yields consistent results for both the two separate autocorrelation sets as well as the cross-correlation set of curves. The obtained diffusion coefficient is ca. $10 \%$ larger than the value obtained from the $z$-scan analysis with neglected adsorption/desorption kinetics. Remarkably, as shown by the values listed in Table 1, only $3 \%$ of all molecules are bound to the surface on average at each time. This also explains why we do not observe a long-time decrease in fluorescence intensity due to photobleaching because there is a continuous exchange of fluorescent molecules in the focal region by the large fraction of fast diffusing molecules. However, this small fraction has a profound influence on the fit performance and the extracted diffusion coefficients, due to the extended time span these molecules remain within the detection region.

\section{Conclusion}

We have developed a new experimental method, 2-focus FCS with overlapping foci, for measuring absolute diffusion coefficients in planar membranes, and applied this method for measuring diffusion of labeled lipids in supported bilayers. We compared our method with the $z$-scan technique and analysis developed by Martin Hof and his group. ${ }^{9}$ Although the $z$-scan analysis seemed to fit the data quite well, the 2-focus method revealed a strong influence of rare adsorption/desorption events of labeled lipids to the support, although only ca. $3 \%$ of all lipids occur to be adsorbed at any time. We developed a theoretical model that takes into account the observed adsorption and desorption kinetics, and applied it to the measured data. We found a very slow adsorption rate constant of the order of 10/s and a desorption kinetics that is by more than 1 magnitude slower (which is probably rather photobleaching than desorption). The slow kinetic rates make accurate FCS measurements rather difficult, but as shown here, adsorption/desorption can be important when aiming at accurate diffusion studies in supported bilayers.

Acknowledgment. I.v.d.H., T.D. and J.E. are much obliged to Benjamin Kaupp for his generous support of our work and for the financial support by the Deutsche Forschungsgemeinschaft (grants EN 297/7, KO 948/6), and the Deutsche Volkswagenstiftung (grant I/79 247) is gratefully acknowledged. A.B and M.H. thank the Grant Agency of the Czech Republic (via 203/ $05 / 2308$ ) for the financial support.

LA061389S 